\title{
Amyloidosis-a rare but mystifying differential of urothelial cell carcinoma of urinary bladder: a case
} report

\begin{abstract}
Haematuria either gross or microscopic has always worried the urologist as it raises the suspicion of malignancy in the genito-urinary tract (GUT). Benign causes of haematuria include inflammation, calculus, infection and trauma to the GUT. Amyloidosis is a very rare but benign cause of haematuria. The important issue is that it simulates transitional cell carcinoma of urinary bladder in presentation, imaging, endoscopic and gross appearance. However the treatment plan and prognosis of both condition is different ranges from various bladder preservation methods to radical cystectomy. Thus an amyloidosis is an important differential of carcinoma urinary bladder that to be should be consider in patients with bladder mass.
\end{abstract}

Keywords: amyloidosis, carcinoma of urinary bladder, haematuria, urinary bladder
Volume 7 Issue 2 - 2019

\author{
Mahendra Pal,Abhay Chaudhary, V Srinivas \\ Hinduja Hospital \& Medical Research Centre, Homi Bhabha \\ National Institute, India
}

\begin{abstract}
Correspondence: Mahendra Pal, Associate professor, University, HBNI, P.D. Hinduja Hospital \& Medical Research Centre, SVS Rd, Asavari, Mahim, Mumbai, Maharashtra 4000I6,
\end{abstract} Tel 022-24449199, Email mahen I 197@gmail.com

Received: February 22, 2019 | Published: April 05, 2019

\section{Keymessage}

Amyloidosis of urinary bladder though uncommon, mimics carcinoma of bladder clinically. Management of amyloidosis of bladder is less aggressive than carcinoma and thus warrants careful histo-pathological examination of resected tissue to avoid unnecessary overtreatment and complications in these patients.

\section{Introduction}

An elderly patient presenting with gross haematuria should be suspected to have urological cancer until proven otherwise. ${ }^{1}$ Amyloidosis rarely occurs in genito urinary tract (GUT). ${ }^{1}$ Around 200 cases have been reported in the urology literature so far. ${ }^{2,3}$ Urinary bladder (UB) is the most common genito-urinary organ to be affected by amyloidosis. ${ }^{3}$ It can present with symptoms similar to carcinoma of UB (CUB) and simulate transitional cell carcinoma (TCC) on radiographic and cystoscopic examination. ${ }^{1}$ Herein, we report a case of primary UB amyloidosis and discuss the available diagnostic and treatment options.

\section{Case report}

A 60 year old male was referred to us for complaints of intermittent painless gross haematuria since 18 months associated with passage of blood clots of variable size. Physical examination was within normal limits and past medical history was non contributory. Haematological and coagulation parameters were within normal limits. Urine analysis showed several WBCs, RBCs with mild albuminuria. The CECT scan of abdomen and pelvis revealed mild right hydronephrosis and an irregular UB wall thickening with few polypoidal growths along its right supero-lateral aspect (Figure 1). On cystoscopy, few papillary growths were observed, largest being $2 \mathrm{~cm}$, at right lateral wall involving the right ureteric orifice. Rest of the bladder mucosa appeared irregular, thickened and edematous. The growth was resected transurethral and random biopsies were taken. Histopathology of the resected tissue revealed abundant deposition of acellular, eosinophlic material beneath the epithelial lining with large amount being deposited in the vessels wall with no tumour cells. Congo red staining and apple-green birefringence in polarized light confirmed the diagnosis of amyloidosis (Figure 2A, 2B). Post-operative recovery remained uneventful and the patient was asymptomatic with no recurrence in bladder on repeated cystoscopy after two year of follow up.

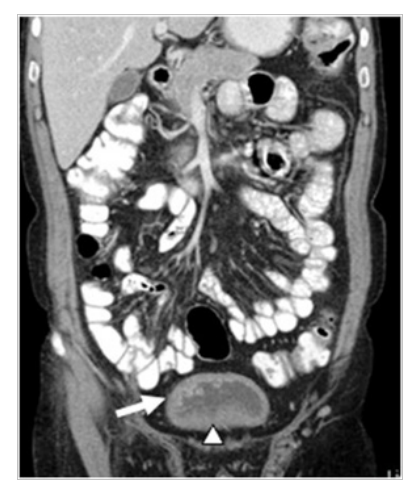

Figure I CECT Showing Bladder Wall Thickening At Base (Arrow Head) And Polypoidal Growths At Right Lateral Wall (Arrow).
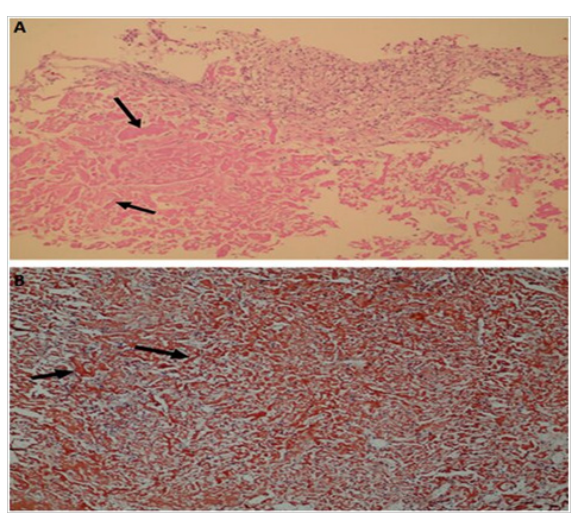

Figure 2 A myloid Deposition (A) In BladderWall (Arrows) 100x, H \& E Stain, (B) Congo Red Stain(Arrow) I00X. 


\section{Discussion}

Amyloidosis is a pathological process characterized by extracellular deposition of proteinaceous material in tissues and organs. ${ }^{1,3}$ It is known to affect cardiac muscle, striated muscle, adipose tissue and gastrointestinal tract and is noted more commonly in patients with a history of chronic inflammatory disorders. ${ }^{3}$ While the aetiology of amyloidosis is unknown, several studies suggest a chronic monoclonal inflammatory response or immunological mechanism. ${ }^{3}$ Amyloidosis of GUT is rare, has varied presentation and is more notably associated with systemic amyloidosis. ${ }^{3}$ It has been reported in the kidney, renal pelvis, ureters, UB and genitals. ${ }^{3}$ Primary amyloidosis of bladder $(\mathrm{PAB})$ is more common than the secondary involvement and simulates CUB. ${ }^{3}$ Both the sexes are affected equally, ${ }^{3}$ the prevalence being higher in elderly population presenting with painless gross haematuria $(75 \%)$ and irritative voiding symptoms $(20 \%))^{2,3}$ A definitive diagnosis can be made by histological identification of amyloid deposits in tissues with Congo-red staining and apple-green birefringence in polarized light. ${ }^{2}$ Prognosis of $\mathrm{PAB}$ is excellent as compared to secondary amyloidosis of bladder ( $\mathrm{SAB})$ as the former does not involve the bladder extensively and is rarely associated with massive haematuria and bladder rupture. ${ }^{3}$ Severe haematuria in SAB may occur due to amyloid deposition in vessel wall that prevents the ruptured vessel to contract. ${ }^{5}$ Management of amyloidosis of GUT is aimed at controlling haematuria hence making endoscopic resection (ER) of the lesion, diagnostic and therapeutic modality of choice. ${ }^{1}$ Symptomatic local recurrence is common (around 50\%) and usually treated by repeat ER. ${ }^{1,2}$ Several other non-invasive and invasive modalities are available for management of amyloidosis of UB as discussed in Table 1.

Table I Treatment options available for amyloidosis of Urinary bladder 1,3-6

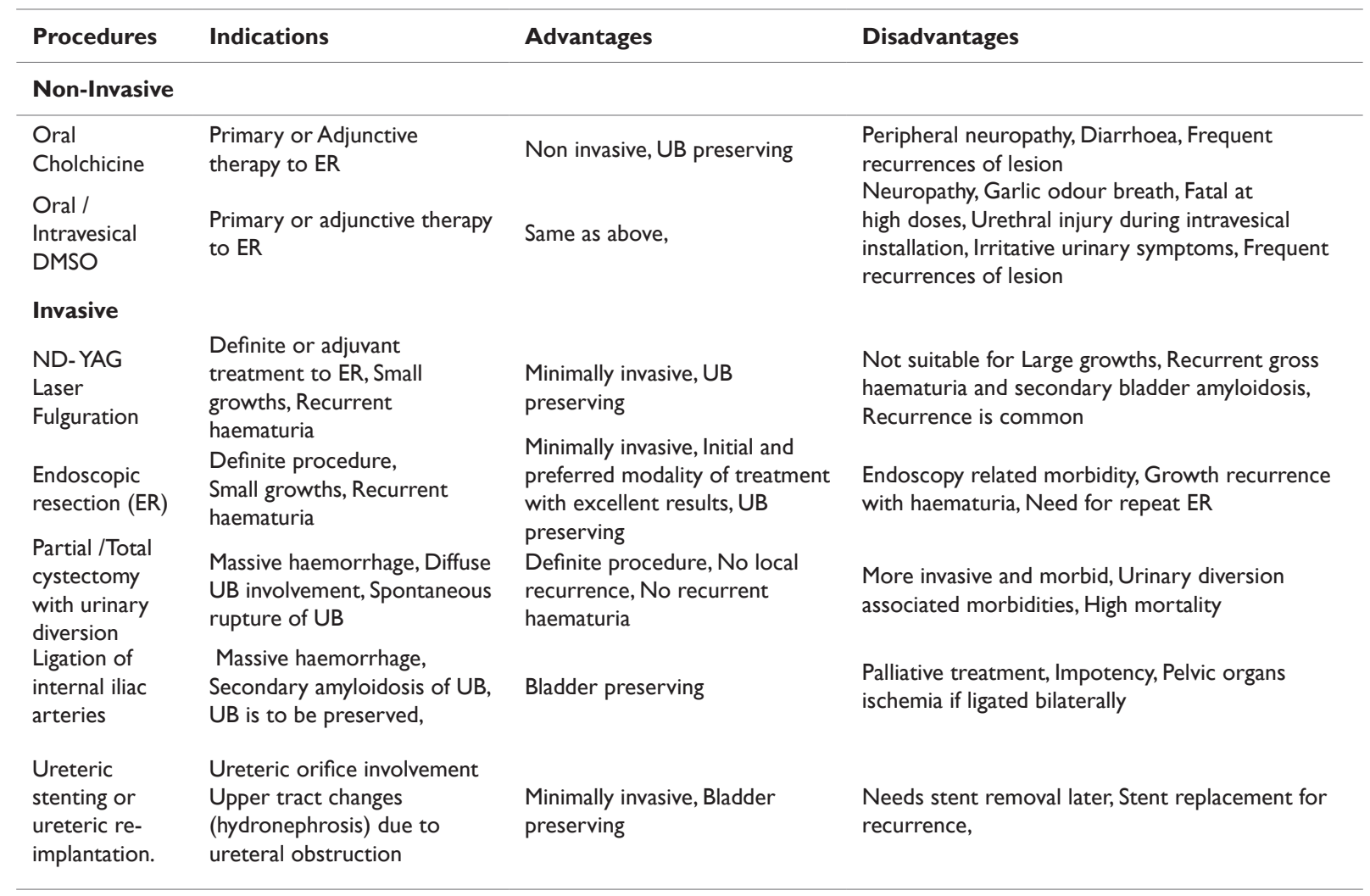

UB, Urinary Bladder; DMSO, Dimethylsulfoxide; ER, Endoscopic resection

\section{Conclusion}

Amyloidosis of UB though a rare cause of irritative voiding symptoms can simulate transitional cell carcinoma of UB on imaging and cystoscopy. Accurate diagnosis remains the key to avoid aggressive treatment of this benign condition. ER is the preferred diagnostic and therapeutic option in these patients with excellent results and can be used in follow-up periods to treat recurrence.

\section{Acknowledgments}

None.

\section{Conflicts of interest}

The authors declared there is no conflict of interest.

\section{References}

1. Yalcin V, Citgez S, Agaoglu I. Localised primary amyloidosis of the urinary bladder: case series of two patients. BMJ Case Rep. 2009.

2. Wilkinson M, Fanning DM, Flood H. Primary bladder amyloidosis. BMJ Case Rep. 2011;20.

3. Agarwal SK, Walmsley BH, Marley NJ. Primary amyloidosis of urinary bladder. Int J Health Sci (Qassim). 1995;88:(3):171P-172P. 
4. Cağlar K, Kibar Y, Tahmaz L, et al. Laser therapy in patient with intractable haemorrhage due to the bladder involvement of systemic amyloidosis. Nephrol Dial Transplant. 2001;16(8):17-24.

5. Chitale S, Morsey Mo, Peat D. Amyloidosis of Lower Genitourinary Tract: A Review. Ralph Web eau-ebu update series. 2007;5;70-76.
6. Jones A, Fergus J. Primary amyloidosis of the urinary bladder and ureters: letter to the editor. Br J Urol. 1998; 81:658. 